\title{
24 synoptic maps 1974-1982 (ascending phase of cycle XXI) of 323 prominence average magnetic fields measured by the Hanle effect
}

\author{
Véronique Bommier
}

LESIA, Observatoire de Paris, CNRS-INSU-UMR8109, UPMC Univ. Paris 06, Université Paris Diderot-Paris 7; 5, Place Jules Janssen, 92190 Meudon, France

\begin{abstract}
The poster was made of 323 average prominence magnetic fields reported on 24 synoptic maps. The paper first resumes the methods for the field derivation, and the different results of the whole program of these second generation Hanle effect observations. From their conclusions, it was possible to derive a unique field vector for each of the 323 prominences. The maps put in evidence a large scale structure of the prominence magnetic field, probably distorted by the differential rotation, which leads to a systematically small angle (on the order of $30^{\circ}$ ) between the field vector and the prominence long axis.
\end{abstract}

Keywords. Prominences, Magnetic Field, Hanle effect, Polarization

\section{Introduction}

The observational discovery of the Hanle effect in prominences is due to Bernard Lyot. Lyot $(1934,1936)$ clearly and repeatedly observed the rotation of the polarization direction, but its first interpretation in terms of the Hanle effect is due to Hyder (1965). Fourty years after Lyot, the observation polarimetric accuracy had increased by one order of magnitude, which has enabled quantitative interpretation of the measurements in terms of magnetic field diagnostics. This required the density matric formalism to be developed in order to generalize the statistical equilibrium equations of the mean atom, to the offdiagonal coherences responsible for the Hanle effect. At the colloquium, it was several times claimed that "only about thirty prominences were completely analyzed" from these observations. On the one hand this is the case with one unique $3 \mathrm{D}$ average field vector determined for only 14 prominences (Bommier et al. 1994), and 18 prominences with 3D field vector determination but ambiguous by Athay et al. (1983), but on the other hand more partial studies, yet leading to important results, were previously led on much larger samples of prominences. In order to help the reader to disentangle the subtly different classes of results, and to follow how the method was developed and was step by step complemented, Sect. 2 is devoted to describe the thread of these Hanle effect studies of the second generation. An unpublished result is given at the end of the section, about the discovery of Inverse Polarity prominences from the analysis of two following days observations, in 1980. The content of the poster, which was made of 24 synoptic maps on which the final magnetic field vector was reported for 323 prominences observed at the Pic-du-Midi during the ascending phase of Cycle XXI, is described in Sect. 3. A unique field vector was obtained for each of these 323 prominences by applying the conclusions of the whole story. 


\section{Thread of the cycle XXI prominence Hanle effect studies}

A campaign of prominence Hanle effect observations was runned at the Pic-du-Midi coronagraph from 1974 to 1982 (Leroy et al. 1977), along the ascending phase of solar cycle XXI. The polarimeter was equipped with a filter and two photomultipliers (Ratier 1975), without any spectral resolution, unnecessary for the Hanle effect. The aperture was a pinhole of 3 arcsec. A dozen of exposures in different locations were typically taken for each prominence, but curiously the polarization was found rather constant (see Fig. 1 of Leroy et al. 1977), so that the polarization was finally averaged over the whole prominence for interpretation to increase the accuracy. The observed line was $\mathrm{He} \mathrm{I}_{3}$, but this was insufficient to fully determine the field vector, because two linear polarization parameters were measured (the linear polarization degree and direction), and three vector coordinates were searched for. Accordingly, two lines at least are necessary for a complete field vector determination. Moreover, these two lines must have different Hanle sensitivity. The Hanle sensitivity of a line is given by $\omega \tau=1$, where $\omega=2 \pi \nu$ where $\nu$ is the Larmor frequency, and $\tau$ is the line upper level lifetime. Typically, a visible permitted line is sensitive to fields of about $10 \mathrm{G}$ (see a table of typical sensitivities in Sahal-Brechot 1981, Table I). $\mathrm{D}_{3}$ is sensitive to $6 \mathrm{G}$, also the typical prominence field.

Two-line observations were runned at the same moment with the experiment Stokes II at Sac. Peak. Stokes II was a spectropolarimeter and the two lines were the two components of the $\mathrm{He} \mathrm{I} \mathrm{D}_{3}$ line. One is made of five unresolved fine-structure components $3 d^{3} D_{3,2,1} \rightarrow 2 p^{3} P_{2,1}$ whereas the other is made of the single $3 d^{3} D_{1} \rightarrow 2 p^{3} P_{0}$. The condition of different sensitivity is fulfilled, and the field vector horizontality (i.e. the field vector lying in the solar horizontal plane) was obtained in 18 (Athay et al. 1983) and 2 (Querfeld et al. 1985) individual prominences. The field horizontality was also statistically visible in the Pic-du-Midi data by superimposing the observed polarization with the horizontal field diagram computed from the new theory by Bommier \& SahalBrechot (1978). The superposition is visible in Fig. 5 of Sahal-Brechot et al. (1977), and the statistical derivation of the field horizontality from this figure is discussed in Bommier (2009).

Once the field horizontality was proven, it was possible to interpret each Pic-du-Midi observation in terms of field strength and azimuth. More than 400 prominences were observed during the whole campaign, which finally result in 323 interpreted prominences by considering only quiescent objects, and by retaining only those prominences that could be identified with a filament observed eight days before (W limb) or after (E limb). 110 prominences were Polar Crown prominences and their magnetic field was presented and discussed in Leroy et al. (1983). In particular, it was obtained that the field strength increases with altitude with a vertical gradient of $+0.5 \times 10^{-4} \mathrm{G} / \mathrm{km}$. The 213 remaining prominences of the sample are lower latitude objects, which have various orientations of their filament with respect to the line-of-sight. Advantage was taken of this variety and of the mirror effect of the symmetry with respect to the line-of-sight, to derive a statistical solution of the fundamental ambiguity (Leroy et al. 1984) from the idea that the angle between the field vector and the filament long axis should be rather constant through the sample. Indeed, the Hanle effect is submitted to the fundamental ambiguity, which originates in the very small atomic size with respect to the radiation wavelength, so that the atom cannot discriminate between the two propagation directions possible along a given ray path. The result is that two field vectors symmetrical with respect to the line-ofsight cannot be discriminated. The symmetry is rigorous when the prominence is located in the plane of the sky, i.e. when the scattering angle is $90^{\circ}$. For different scattering angles, there are two ambiguous field vectors but they are not strictly symmetrical with 


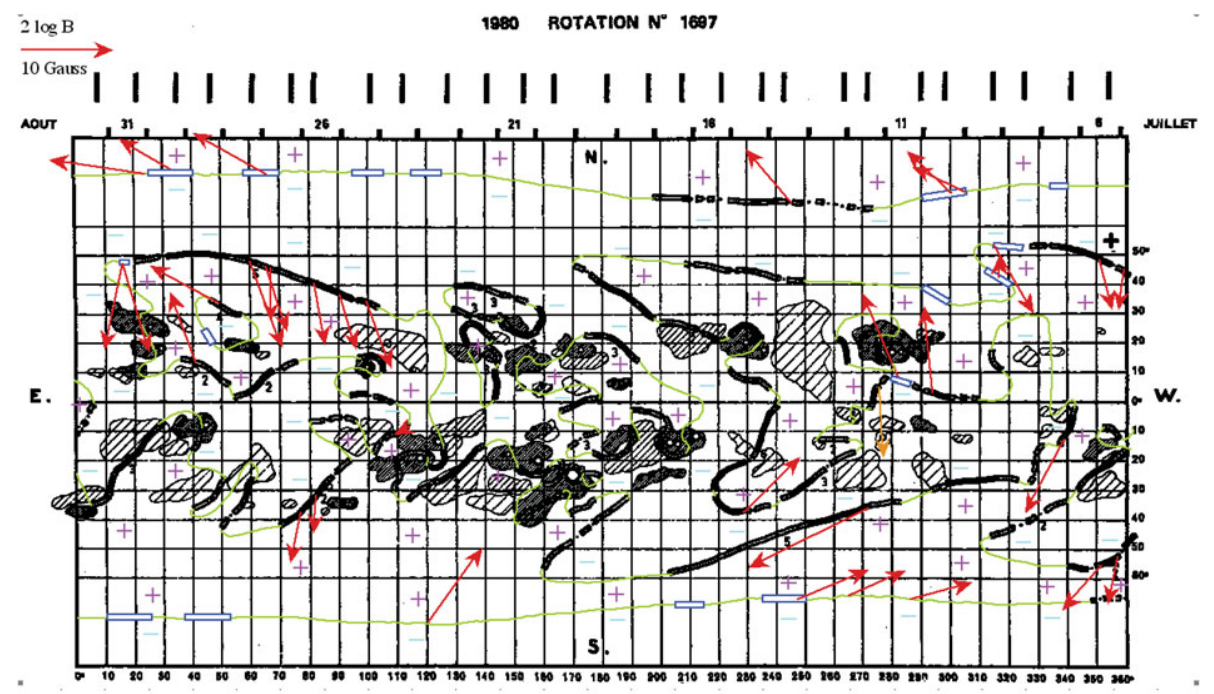

Figure 1. Example of the Meudon Observatory synoptic map of Carrington Rotation 1697 (July 1980), with prominence average magnetic field vectors superimposed. Photospheric neutral lines and polarities, and Polar Crown prominences have been added taken from the McIntosh map.

respect to the line-of-sight. The statistical resolution led to $75 \%$ of prominences of the Inverse Polarity type (Kuperus-Raadu type), whereas the $25 \%$ remaining ones were of the Normal Polarity type (Kippenhahn-Schlüter type).

Two-lines observations were also runned during the last years of the campaign, after the modification of the polarimeter to enable multiline observations. Hydrogen $\mathrm{H} \beta$ or $\mathrm{H} \alpha$ were observed together with $\mathrm{D}_{3}$. Hydrogen $\mathrm{H} \beta$ is optically thin in prominences, and its Hanle sensitivity is different from the $\mathrm{D}_{3}$ one, so that the full vector was determined and the field horizontality was again obtained in 18 prominences (Bommier et al. 1986b). Four polarization parameters were measured with the two lines, and the field vector is three coordinates only, so that a parameter remained free, which was devoted to the electron density determination via the collisional depolarization. The electron density was found to range from $10^{9}$ to $4 \times 10^{10} \mathrm{~cm}^{-3}$ in these 18 prominences (Bommier et al. 1986a). $\mathrm{H} \alpha$ is optically thick and a model of its radiative transfer was developed by Landi Degl'Innocenti et al. (1987). The effect of the optical thickness is that there is a non-negligible incident radiation along the filament axis, and that the underlying photospheric radiation is differentially absorbed on both sides of the prominence. The scattering geometry is then much more complicate than in the optically thin case, and the result is that two ambiguous field vectors are derived, which do not have the same symmetry as in the optically thin case. Advantage may then be taken of this difference to solve the fundamental ambiguity. This was done in 14 prominences in Bommier et al. (1994), leading to 12 Inverse and only 2 Normal Polarity prominences. Besides, the ensemble of results on the prominence magnetic field is summarized in Table I of that paper, with in particular the angle of about $35^{\circ}$ found between the field vector and the filament long axis.

A third method was explored for solving the ambiguity, which is the comparison of the results of prominences observed on two following days. This was investigated by Bommier et al. (1981) and found very efficient, but the result remained unpublished because too surprising at that moment. A large majority of Inverse Polarity prominences were found in the sample of about twenty analyzed prominences. 


\section{Content of the presented poster}

Once taken the results on the field horizontality and the prominence polarity, it is possible to infer a unique field vector for each of the 323 prominences observed at the Pic-du-Midi, and to plot this field vector on the synoptic maps. This was the object of the poster, where 24 synoptic maps were presentend that contained the 323 prominences. One of those is given here in Fig. 1. In the few cases where the two ambiguous field vectors were pointing on the same side of the filament and then were of the same polarity so that the ambiguity could not be solved by the polarity law, the ambiguity was removed by selecting the one of the two vectors that had the same orientation of the field component along the filament, as another prominence observed along the same neutral line. A more detailed presentation of this systematic ambiguity solution is presented in Bommier (2009), with figures.

On the map given in example in Fig. 1, the continuity of the filament long axis field component is visible along the neutral line, and in addition its sign changes when crossing successive neutral lines from north to south, or from east to west. It appears also that the prominence field is in accordance with alignment along a north-south general line distorted by the differential rotation. Such results were visible in each of the 24 maps.

\section{Conclusion}

For page number limit reasons, it was not possible to attach the 24 synoptic maps to this publication. This will be the object of a forthcoming paper. Nevertheless the large scale structure of the prominence magnetic field, which seems to be strongly linked to the one of the neutral line, is already visible in the example map given in Fig. 1 of this paper. The small angle (on the order of $30^{\circ}$ ) between the field vector and the prominence long axis appears as systematic. It could be the result of a north-south general field line distorted by the differential rotation. The smallness of the angle leads to the necessity of 3D MHD modeling. The third generation of Hanle effect observations will be spatially resolved prominence observations. They have presently begun, but the preliminary investigation by Leroy et al. (1977) in their Fig. 1 displays a rather homogeneous field, which seems to contradict the highly structured aspect of the $\mathrm{H} \alpha$ observations. This feature presently remains to be explained.

\section{References}

Athay, R. G., Querfeld, C. W., Smartt, et al. 1983, Solar Phys., 89, 3

Bommier, V. 2009, in Lecture Notes in Physics, Berlin Springer Verlag, Vol. 765, 231-259

Bommier, V., Landi Degl'Innocenti, E., Leroy, J.-L., \& Sahal-Brechot, S. 1994, Solar Phys., 154, 231

Bommier, V., Leroy, J. L., \& Sahal-Brechot, S. 1986a, Astron. Astrophys., 156, 90

Bommier, V. \& Sahal-Brechot, S. 1978, Astron. Astrophys., 69, 57

Bommier, V., Sahal-Brechot, S., \& Leroy, J. L. 1981, Astron. Astrophys., 100, 231

Bommier, V., Sahal-Brechot, S., \& Leroy, J. L. 1986b, Astron. Astrophys., 156, 79

Hyder, C. L. 1965, Astrophys. J., 141, 1374

Landi Degl'Innocenti, E., Bommier, V., \& Sahal-Brechot, S. 1987, Astron. Astrophys., 186, 335

Leroy, J. L., Bommier, V., \& Sahal-Brechot, S. 1983, Solar Phys., 83, 135

Leroy, J. L., Bommier, V., \& Sahal-Brechot, S. 1984, Astron. Astrophys., 131, 33

Leroy, J. L., Ratier, G., \& Bommier, V. 1977, Astron. Astrophys., 54, 811

Lyot, B. 1934, Compt. Rend. Acad. Sci., 198, 249

Lyot, B. 1934, Compt. Rend. Acad. Sci., 202, 392

Querfeld, C. W., Smartt, R. N., Bommier, V., et al. 1985, Solar Phys., 96, 277

Ratier, G. 1975, Nouvelle Revue d'Optique, 6, 149

Sahal-Brechot, S. 1981, Space Sci. Rev., 29, 391

Sahal-Brechot, S., Bommier, V., \& Leroy, J. L. 1977, Astron. Astrophys., 59, 223 